\title{
Top FCNC interactions through dimension-six four-fermion operators at the electron proton collider
}

\author{
Wei $\mathrm{Liu}^{1, *}$ and Hao Sun ${ }^{2, \dagger}$ \\ ${ }^{1}$ University College London, Gower Street, London WC1E 6BT, United Kingdom \\ ${ }^{2}$ Institute of Theoretical Physics, School of Physics, Dalian University of Technology, \\ No. 2 Linggong Road, Dalian, Liaoning, 116024, People's Republic of China
}

(Received 10 April 2019; published 10 July 2019)

\begin{abstract}
We investigate the flavor changing neutral currents (FCNC) generated by dimension-six four-fermion operators at the Large Hadron-Electron Collider ( $\mathrm{LHeC})$ proposed at CERN in an effective approach. This is performed by Monte Carlo analysis at the full detector level, as the background is successfully reduced by using an invariant mass scheme to require the final states to reconstruct the mass (transverse mass) of the top quark ( $W$ boson). Our analysis shows that the future electron proton colliders like the LHeC can probe competitive limits for the top FCNC dimension-six four-fermion operators such as $C_{l q}^{(1) e e 31}<0.0647$, $C_{l u}^{e e 31}<0.109, C_{\text {lequ }}^{(1) e e 31}<0.217$ and $C_{\text {lequ }}^{(3) e e 31}<0.0209$ in the Warsaw basis.
\end{abstract}

DOI: $10.1103 /$ PhysRevD.100.015011

\section{INTRODUCTION}

Although the Standard Model (SM) has been tested to a great precision, it is commonly considered as an effective field theory which is applicable up to a certain scale $\Lambda$. Following this, several works [1-7] have appeared on the top quark, the most important, heaviest particle known up to the electroweak scale, based on the dimension-six operators [8-10] of the Standard Model effective field theory (SMEFT) at the LHC. Among these operators, the ones which introduce flavor changing neutral currents (FCNC) are very sensitive to new physics as they are extremely suppressed by the Glashow-Iliopoulos-Maiani (GIM) mechanism [11] in the SM. Meanwhile, a proposed electron proton collider called the Large Hadron-Electron Collider ( $\mathrm{LHeC}$ ) $[12,13]$ serves as one of the possible options in the post-LHC era. As the incoming protons are produced from the LHC beam, the high energy makes it suitable for studies of the top quark. Moreover, the asymmetric beams make $\mathrm{LHeC}$ much more suitable for probing top FCNC operators, in particular, leptons and quarks. Such dimension-six operators can include gauge bosons; as it introduces much more complex processes and backgrounds, we focus on the operators including four

\footnotetext{
wei.liu.16@ucl.ac.uk

†haosun@mail.ustc.edu.cn; haosun@dlut.edu.cn
}

Published by the American Physical Society under the terms of the Creative Commons Attribution 4.0 International license. Further distribution of this work must maintain attribution to the author(s) and the published article's title, journal citation, and DOI. Funded by SCOAP ${ }^{3}$. fermions at the $\mathrm{LHeC}$, such as the ones which can produce $e^{-} p \rightarrow e^{-} t$ process.

As the hints of lepton flavor universality violation $b \rightarrow$ sl $\ell$ and $b \rightarrow c \tau \nu$ [14-16] at the LHCb received much attention, our interest is mainly reinterpreted through the CKM matrix $[17,18]$. A summary of the current limits on different corresponding parameters at the LHC can be found in Ref. [5], which gives the limits on the Wilson coefficients of the top involved four-fermion operators including two quarks and two leptons at $\mathcal{O}(0.1)$ assuming $\Lambda=1 \mathrm{TeV}$. Such studies have also been carried out in the $e^{-} e^{+}$colliders [19-24], yielding the most stringent limits on some Wilson coefficients such as $C_{l q}^{(3) e e 33}$ at $\mathcal{O}(0.01)$ assuming a relatively larger $\Lambda=10 \mathrm{TeV}$ [21]. This is obtained by assuming the experimental uncertainty to be as high as $30 \%$. However, at the $\mathrm{LHeC}$, most effort is put into dimension-four FCNC operators [25-30]. The exact process $e^{-} p \rightarrow e^{-} t$ has been considered in a recent paper [25] while focusing on anomalous $\mathrm{Ztq}$ couplings. Apart from these, a study at the $\mathrm{LHeC}$ about the dimension-six operator containing the Higgs boson has been carried out in Ref. [31]. Reference [32,33] study a similar quarkquark-lepton-lepton $(Q Q L L)$ operator for a different generation which does not contain top quarks but includes Majorana neutrinos. A similar study considered a neutrino in the final state rather than an electron, while an extra $t b W$ interaction has been considered with a focus on non-flavor changing operators in Ref. [34].

In this paper, we focus on the operators in the basis of dimension-six SMEFT which can generate a single top production $e^{-} p \rightarrow e^{-} t$ process at the $\mathrm{LHeC}$, i.e., fourfermion operators at the 1113 generation. We select the 
scale factor $\Lambda$ in the Wilson coefficient to be $1 \mathrm{TeV}$ for comparison. Particularly, we reconstruct the top in the final states using the leptonic channel of its decay, $b, e^{-}$and $E_{T}^{\text {miss }}$ regrading the current capability of the full detector level simulation of the $\mathrm{LHeC}$. By putting cuts on the reconstructed top mass and the transverse mass of the $W$ boson, a relatively high significance $S / \sqrt{B}$ is obtained.

The paper is organized as follows: In Sec. II, we briefly review the dimension-six Standard Model effective field theory and summarize the relevant operators for the process $e^{-} p \rightarrow e^{-} t$. Following this, we simulate the cross section of this signal process depending on the corresponding Wilson coefficient, analyze the main background at the $\mathrm{LHeC}$, and put kinematical cuts on them to reduce the background and finally obtain the sensitivities in Sec. III. We summarize the paper and give the conclusions in Sec. IV.

\section{DIMENSION-SIX FCNC FOUR-FERMION CONTACT OPERATORS IN SMEFT}

\section{A. Relevant operators}

When physics beyond the SM is present at scales $(\Lambda)$ larger than the electroweak scale, the SM can be extended into an EFT. The so-called SMEFT, including the same symmetric group $S U(3) \times S U(2) \times U(1)$ of the $\mathrm{SM}$, defined by a power counting expansion in the ratio of the scales, extends the SM with higher dimensional operators $\mathcal{Q}_{i}^{(d)}$ of mass dimension $d$. The Lagrangian is expressed as

$\mathcal{L}_{\mathrm{SMEFT}}=\mathcal{L}_{\mathrm{SM}}^{(4)}+\sum_{k} \frac{C_{k}^{(5)}}{\Lambda} \mathcal{Q}_{k}^{(5)}+\sum_{k} \frac{C_{k}^{(6)}}{\Lambda^{2}} \mathcal{Q}_{k}^{(6)}+\mathcal{O}\left(\frac{1}{\Lambda^{3}}\right)$

where $\mathcal{L}_{\mathrm{SM}}^{(4)}$ is the SM Lagrangian and $C_{k}^{(d)}$ stands for the corresponding dimensionless coupling constants (Wilson coefficients). The dimension-five terms vanish if baryon and lepton number conservation is imposed, and the dimensionsix terms, which we concentrate on, are $\sum_{k} \frac{C_{k}^{(6)}}{\Lambda^{2}} \mathcal{Q}_{k}^{(6)}$.

A complete set of all allowed dimension-six operators is actually quite large. However, not all operators, obeying the required symmetries of the SM Lagrangian, are independent. They are related by the equations of motion and also by Fierz transformations. Therefore, the total number of operators can be reduced to a minimum set of independent ones. For simplification, they can be classified into three different groups: strong, electroweak, and four-fermion operators [35].

In our present paper, we concentrate on the four-fermion operators, where the complete nonredundant set of fourfermion operators are shown in [8] and referenced in the so-called "Warsaw basis." Here the operators should be supplemented with generation indices of the fermion fields whenever necessary, e.g., $Q_{q q}^{(1)} \rightarrow Q_{q q}^{(1) p r s t}$. Here $p, r, s$, $t=1,2,3$ are generation indices, (1) and (3) are isospin indices, and (1) and (8) are color indices. Excluding the five B-violating operators, whose effects must be strongly suppressed to respect proton decay bounds, we have 59 independent operators. In fact, one can relax the flavor assumptions and allow all possible flavor combinations to be independent operators. This increases the operator set to 2499 operators. The set of four-fermion operators can be further reduced when only specific processes are studied. For example, at electron proton (ep) colliders, ep collision can be used to study four-fermion interaction which involves two leptons and two quarks. They may therefore be sensitive to $Q_{l q}^{(1)}, Q_{l q}^{(3)}, Q_{\ell u}, Q_{e u}, Q_{\text {lequ }}^{(1)}$ and $Q_{\text {lequ }}^{(3)}$ which are given as

$$
\begin{aligned}
\mathcal{O}_{l q}^{(1) p r s t} & =\left(\bar{l}_{p} \gamma_{\mu} l_{r}\right)\left(\bar{q}_{s} \gamma^{\mu} q_{t}\right), \\
\mathcal{O}_{l q}^{(3) p r s t} & =\left(\bar{l}_{p} \gamma_{\mu} \tau^{I} l_{r}\right)\left(\bar{q}_{s} \gamma^{\mu} \tau^{I} q_{t}\right), \\
\mathcal{O}_{\text {lu }}^{\text {prst }} & =\left(\bar{l}_{p} \gamma_{\mu} l_{r}\right)\left(\bar{u}_{s} \gamma^{\mu} u_{t}\right), \\
\mathcal{O}_{\text {eq }}^{\text {prst }} & =\left(\bar{e}_{p} \gamma_{\mu} e_{r}\right)\left(\bar{q}_{s} \gamma^{\mu} q_{t}\right), \\
\mathcal{O}_{\text {eu }}^{\text {prst }} & =\left(\bar{e}_{p} \gamma_{\mu} e_{r}\right)\left(\bar{u}_{s} \gamma^{\mu} u_{t}\right), \\
\mathcal{O}_{\text {lequ }}^{(1) p r s t} & =\left(\bar{l}_{p} e_{r}\right) \epsilon\left(\bar{q}_{s} u_{t}\right), \\
\mathcal{O}_{\text {lequ }}^{(3) p r s t} & =\left(\bar{l}_{p} \sigma_{\mu \nu} e_{r}\right) \epsilon\left(\bar{q}_{s} \sigma^{\mu \nu} u_{t}\right) .
\end{aligned}
$$

The notation employed in this section follows that of Ref. [8], with flavor indices labeled by prst; left-handed fermion doublets denoted by $q, l$; right-handed fermion singlets by $u$, $d, e$; and the antisymmetric $S U(2)$ tensor by $\varepsilon \equiv i \tau^{2}$, where $\tau^{I}$ are the Pauli matrices.

Furthermore, we consider the single top production effects at ep colliders, so we should use a set of dimension-six fourfermion operators involving at least one top quark and at least one electron. Our discussion exclusively concerns processes involving at least a top quark. Only operators involving such a particle are considered. Other operators affecting the considered processes are assumed to be well constrained by measurements in processes that do not involve top quarks. This assumption may not always be justified, and explicit checks should be performed.

\section{B. Current limits}

There are no direct limits on the top related flavor changing four-fermion operators so far. However, various studies about four-fermion operators can be applied through the measurement of CKM matrix elements. Indirect limits from low-energy observables, including $B$ decays, dilepton production, electric dipole moments, $C P$ asymmetries, proposed $e^{-}, e^{+}$colliders and future $\mathrm{LHC}$ upgrades such as HL-LHC as well as HE-LHC [36], can apply to the operators mentioned above, here we only introduce the limits available at current experiments as discussed in the following subsections. We follow a similar 
procedure as in Ref. [5], but instead of assuming the (33) element to be the only effective one, we assume an offdiagonal (13) or (23) element for top flavor changing.

\section{Limits from B physics}

Consider the charged-current $b \rightarrow c e_{i} \bar{\nu}_{j}$ :

$$
\begin{aligned}
H_{\mathrm{eff}}^{b \rightarrow c e \overline{\nu_{j}}}= & -\frac{2}{\nu^{2}} V_{c b}\left(\left(\delta^{i j}+c_{V_{L}}^{i j}\right)\left(\overline{c_{L}} \gamma^{\mu} b_{L}\right)\left(\overline{e_{L}^{i}} \gamma^{\mu} \nu_{L}^{j}\right)\right. \\
& \left.+c_{S_{R}}^{i j}\left(\overline{c_{L}} b_{R}\right)\left(\overline{e_{R}^{i}} \nu_{L}^{j}\right)+\text { H.c. }\right)
\end{aligned}
$$

where $\nu \approx 246 \mathrm{GeV}$. The tree-level matching to the SMEFT is given by $[37,38]$

$$
\begin{gathered}
c_{V_{L}}^{i j}=-\frac{\nu^{2}}{\Lambda^{2}}\left(\frac{\Sigma_{k} V_{c k} C_{l q}^{(3) i j 3 k}}{V_{c b}}\right)+\frac{\nu^{2}}{\Lambda^{2}}\left(\frac{\Sigma_{k} V_{c k} C_{\phi q}^{(3) k 3}}{V_{c b}}\right) \delta^{i j}, \\
c_{S_{R}}^{i j}=\frac{\nu^{2}}{\Lambda^{2}}\left(\frac{\Sigma_{k} V_{c k} C_{l q}^{(3) i j 3 k}}{V_{c b}}\right) .
\end{gathered}
$$

Assuming that only the relevant operators are nonvanishing, we get

$$
c_{V_{L}}^{i j}=-\frac{\nu^{2}}{\Lambda^{2}}\left(\frac{V_{c d} C_{l q}^{(3) i j 31}}{V_{c b}}\right)
$$

or

$$
c_{V_{L}}^{i j}=-\frac{\nu^{2}}{\Lambda^{2}}\left(\frac{V_{c s} C_{l q}^{(3) i j 32}}{V_{c b}}\right) .
$$

For example, in Refs. [5,39]

$$
\left|1+c_{V_{L}}^{\tau \tau}\right|^{2}=1.237 \pm 0.053
$$

Applying $\quad c_{V_{L}}^{\tau \tau}=-\frac{\nu^{2}}{\Lambda^{2}}\left(\frac{V_{c b} C_{l q}^{(3) \tau \tau 33}}{V_{c b}}\right)$, we obtain $C_{l q}^{(3) \tau \tau 33}=$ $-1.85 \pm 0.40$ which agrees with the result shown in Ref. [5]. Thus, similar results can be obtained by translation by dividing the CKM matrix $\frac{V_{c d}}{V_{c b}}$ or $\frac{V_{c s}}{V_{c b}}$, with the translated results of Ref. [18] shown in Table I.

TABLE I. Current limits on the relevant operators when $\Lambda=$ $1 \mathrm{TeV}$ for 31 (32) or 13 (23) generations.

\begin{tabular}{lc}
\hline \hline$C_{l q}^{(1)}, C_{l q}^{(3)}$ & $C_{l q}^{(3) e e 31} \in[-0.10,0.04]([-0.024,0.0093])[18]$, \\
& $C_{l q}^{(1) e e 31}+C_{l q}^{(3) e e 31} \in[-0.059,0.037]([-0.014$, \\
& $0.0085])[17]$, \\
$C_{e q}$ & $C_{e q}^{e e 31} \in[-0.044,0.051]([-0.010,0.012])[17]$ \\
$C_{l u}$ & No current limits \\
$C_{e u}$ & No current limits \\
$C_{l e q u}^{(1)}$ & No current limits \\
$C_{l e q u}^{(3)}$ & No current limits \\
\hline \hline
\end{tabular}

\section{Limits from high- $p_{T}$ dilepton searches}

The effective operators can be strictly limited by the high-energy tail of $2 \rightarrow 2$ scattering processes such as $q q \rightarrow l l$ in Ref. [17]. Such limits are only valid if the maximal center-of-mass energy $E_{\max }$ is much lower than the massive mediators $M_{N P}$ which have been integrated out. The limits on the flavor changing top operators can be obtained by using the same method provided in the former subsection; the results are shown in Table I.

\section{Limits from electric dipole moments}

As electric dipole moments have been constrained very strictly, it can be sensitive to new physics including fourfermion operators. At the one-loop level, the electric dipole moment can be induced by a single top loop. Thus, at the one-loop level, only same flavor quarks contribute to this process, which is not applicable for the favor changing operators.

\section{NUMERICAL RESULTS}

\section{A. Simulation at the $\mathrm{LHeC}$}

For the simulation of the collider phenomenology, we use FeynRules [40] to extract the Feynman rules from the Lagrangian. The model is generated into Universal FEYNRULES Output (UFO) files [41] from Ref. [5] and then fed into the Monte Carlo event generator MADGRAPH@NLO [42] for the generation of event samples. In order to estimate the event rate at the parton level for the signal and backgrounds, we apply the following basic preselections:

$$
\begin{aligned}
\mathrm{p}_{\mathrm{T}}^{\mathrm{b}, \mathrm{j}, \ell} & >5 \mathrm{GeV}, \quad\left|\eta^{\mathrm{b}, \mathrm{j}(\ell)}\right|<8(5), \\
\Delta \mathrm{R}\left(\mathrm{k}_{1} \mathrm{k}_{2}\right) & >0.4, \quad \mathrm{k}_{1} \mathrm{k}_{2}=\mathrm{jj}, \mathrm{j} \ell, \mathrm{jb}, \mathrm{bb}, \mathrm{b} \ell,
\end{aligned}
$$

where $\Delta \mathrm{R}=\sqrt{\Delta \Phi^{2}+\Delta \eta^{2}}$ is the separation in the rapidity $(\eta)$-azimuth $(\Phi)$ plane; $\mathrm{p}_{\mathrm{T}}^{\mathrm{j}, \mathrm{b}, \ell}$ are the transverse momenta of jets, $b$ jets, and leptons; $\eta$ is the pseudorapidity for the corresponding particles; and the cuts are only put here to create initial samples as we will pass the samples to more delicate cuts for the $\mathrm{LHeC}$ detectors introduced below. The cuts are defined in the lab frame. We pass the generated parton-level events to PythiA6.4 [43] which handles the initial and final state parton shower, hadronization, heavy hadron decays, etc. DeLPHES3.4.1 [44] is used for detector simulation with HEPMC [45] file as the input. The detector is assumed to have a cylindrical geometry comprising a central tracker followed by an electromagnetic and a hadronic calorimeter. The forward and backward regions are also covered by a tracker, and an electromagnetic and a hadronic calorimeter. The angular acceptance for charged tracks in the pseudorapidity range of $-4.3<\eta<4.9$ and the detector performance in terms of momentum and energy resolution of electrons, muons, and jets are based on 
the $\mathrm{LHeC}$ detector design $[13,46]$ (details here are taken from our former paper [47]). For our simulation, a modified PythIA version tuned for the ep colliders and the delphes card files for the LHeC detector configurations [48] are used. We use NN23LO1 $[49,50]$ parton distribution functions for all event generations. The factorization and renormalization scales for both the signal and the background simulations are done with the default MadGraph5 dynamic scales. Anti- $k_{t}$ algorithm is adopted using the jet definition. The other parameters, like the $W$-boson and topquark mass, are chosen to be $M_{W}=80.385 \mathrm{GeV}$ [51] and $M_{t}=172.32 \mathrm{GeV}$. The collision energies are chosen to be a $60 \mathrm{GeV}$ electron beam and a $7 \mathrm{TeV}$ proton beam as proposed.

\section{B. Signal production at the $\mathrm{LHeC}$}

We start with

$$
e^{-}\left(p_{1}\right)+u\left(p_{2}\right) \rightarrow e^{-}\left(p_{3}\right)+t\left(p_{4}\right) .
$$

The process involving the second generation can be calculated analogously, and the contribution of the third generation is negligible. The Feynman diagram for the process is shown in Fig. 1.

After Fierz transformation the part of the Lagrangian containing the above four-Fermi operators can be parametrized as follows [19]:

$$
\begin{aligned}
\mathcal{L}_{\text {tuee }}= & \frac{1}{\Lambda^{2}} \sum_{i, j=L, R}\left[V_{i j}\left(\bar{e} \gamma_{\mu} P_{i} e\right)\left(\bar{t} \gamma^{\mu} P_{j} u\right)+S_{i j}\left(\bar{e} P_{i} e\right)\left(\bar{t} P_{j} u\right)\right. \\
& \left.+T_{i j}\left(\bar{e} \sigma_{\mu \nu} P_{i} e\right)\left(\bar{t} \sigma^{\mu \nu} P_{j} u\right)\right]
\end{aligned}
$$

where $P_{L, R}=\left(1 \mp \gamma_{5}\right) / 2, \sigma^{\mu \nu}=\frac{i}{4}\left[\gamma^{\mu} \gamma^{\nu}-\gamma^{\nu} \gamma^{\mu}\right]$, and we express these vectorlike $\left(V_{i j}\right)$, scalarlike $\left(S_{i j}\right)$ and tensorlike $\left(T_{i j}\right)$ couplings in terms of the coefficients of the seven four-Fermi operators we mentioned above. Applying Fierz transformation, we get the coefficient for the following terms:

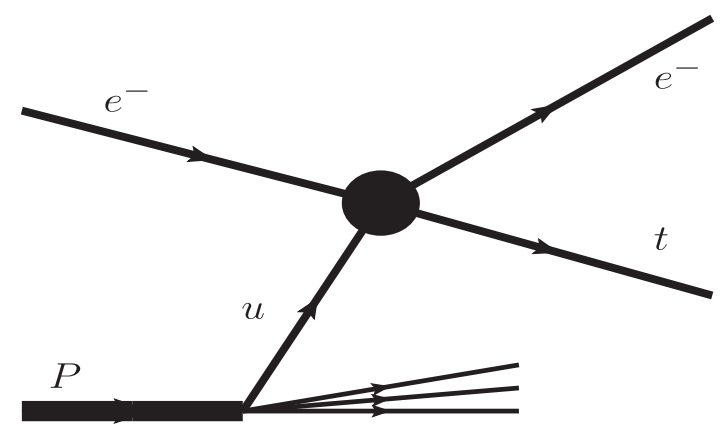

FIG. 1. Signal production through ep collision.

$$
\begin{aligned}
V_{L L} & =C_{\ell q}^{(1)}-C_{\ell q}^{(3)}+C_{\ell q}^{(1) *}-C_{\ell q}^{(3) *}, \\
V_{L R} & =C_{\ell u}+C_{\ell u}^{*}, \quad V_{R L}=C_{q e}+C_{q e}^{*}, \\
V_{R R} & =C_{e u}+C_{\ell u}^{*}, \\
S_{R R} & =-\left(C_{\ell e q u}^{(1)}+C_{\ell e q u}^{(1) *}\right), \\
S_{L L} & =S_{L R}=S_{R L}=0, \\
T_{R R} & =\frac{1}{4}\left(C_{\ell e q u}^{(3)}+C_{\ell e q u}^{(3) *}\right), \\
T_{L L} & =T_{L R}=T_{R L}=0 .
\end{aligned}
$$

The corresponding amplitude can be written as

$\left|\mathcal{M}_{L L}^{V}\right|^{2}=\frac{V_{L L}^{2}}{\Lambda^{4}} 4 \hat{s}\left(\hat{s}-m_{t}^{2}\right), \quad\left|\mathcal{M}_{L R}^{V}\right|^{2}=\frac{V_{L R}^{2}}{\Lambda^{4}} 4 \hat{u}\left(\hat{u}-m_{t}^{2}\right)$,

$\left|\mathcal{M}_{R L}^{V}\right|^{2}=\frac{V_{R L}^{2}}{\Lambda^{4}} 4 \hat{u}\left(\hat{u}-m_{t}^{2}\right), \quad\left|\mathcal{M}_{R R}^{V}\right|^{2}=\frac{V_{R R}^{2}}{\Lambda^{4}} 4 \hat{s}\left(\hat{s}-m_{t}^{2}\right)$,

$\left|\mathcal{M}_{R R}^{S}\right|^{2}=\frac{S_{R R}^{2}}{\Lambda^{4}} \hat{t}\left(\hat{t}-m_{t}^{2}\right)$,

$\left|\mathcal{M}_{R R}^{T}\right|^{2}=\frac{T_{R R}^{2}}{\Lambda^{4}}\left[2 \hat{s}\left(\hat{s}-m_{t}^{2}\right)-\hat{t}\left(\hat{t}-m_{t}^{2}\right)+2 \hat{u}\left(\hat{u}-m_{t}^{2}\right)\right]$,

where $\hat{s}=\left(p_{1}+p_{2}\right)^{2}=\left(p_{3}+p_{4}\right)^{2}, \quad \hat{t}=\left(p_{1}-p_{2}\right)^{2}$, $\hat{u}=\left(p_{1}-p_{3}\right)^{2}$. Thus, we have

$$
\frac{d \hat{\sigma}}{d \Omega}=\frac{1}{64 \pi^{2} \hat{s}} \frac{\hat{s}-m_{t}^{2}}{\hat{s}} \overline{|M|^{2}}
$$

with

$$
\overline{|\mathcal{M}|^{2}}=\frac{1}{4} \sum_{i j=L, R}\left(\left|\mathcal{M}_{i j}^{V}\right|^{2}+\left|\mathcal{M}_{i j}^{S}\right|^{2}+\left|\mathcal{M}_{i j}^{T}\right|^{2}\right) .
$$

The total cross section can be written as

$$
\sigma=\int_{x_{p}^{\min }}^{1} d x_{p} \sum_{q, \bar{q}} f_{q / p}\left(x_{p}, \mu_{f}^{2}\right)\left(\frac{d \hat{\sigma}}{d \Omega}\right) d \Omega
$$

with $x_{p}^{\min }=m_{t}^{2} / s$ and $\hat{s}=x_{p} s$.

As for the decay of the top quark in the $\mathrm{LHeC}$, since the Standard Model $t w b$ coupling is much bigger than the constrained FCNC couplings, the branching ratio for top decays to FCNC processes is considered negligible here, which is also justified in several references [34,52].

The dependence on all independent parameters is simply

$$
\begin{aligned}
\sigma\left(e^{-} p \rightarrow t e^{-}\right) \simeq & 1.63 \cdot\left(V_{L L}^{2}+V_{R R}^{2}\right) \\
& +0.576 \cdot\left(V_{L R}^{2}+V_{R L}^{2}\right) \\
& +0.145 \cdot S_{R R}{ }^{2}+246 \cdot T_{R R}{ }^{2}[\mathrm{pb}] .
\end{aligned}
$$

In the basis of $C$ in the Wilson coefficient, 


$$
\begin{aligned}
\sigma\left(e^{-} p \rightarrow t e^{-}\right) \simeq & 1.63 \cdot\left(\left(C_{l q}^{(1) e e 31}-C_{l q}^{(3) e e 31}+\text { H.c. }\right)^{2}+\left(C_{e u}^{e e 31}+\text { H.c. }\right)^{2}\right)+0.576 \cdot\left(\left(C_{e q}^{e e 31}+\text { H.c. }\right)^{2}+\left(C_{l u}^{e e 31}+\text { H.c. }\right)^{2}\right) \\
& +0.145 \cdot\left(\left(C_{\text {lequ }}^{(1) e e 13}+\text { H.c. }\right)^{2}+\left(C_{l e q u}^{(1) e e 31}+\text { H.c. }\right)^{2}\right)+15.6 \cdot\left(\left(C_{l e q u}^{(3) e e 13}+\text { H.c. }\right)^{2}+\left(C_{l e q u}^{(3) e e 31}+\text { H.c. }\right)^{2}\right)[\mathrm{pb}] .
\end{aligned}
$$

Here we use $\Lambda=1 \mathrm{TeV}$ as the default set. Using analogous methods for other partons, we can obtain the dependence for different generations. In the calculations that follow, we take the condition that only $C_{l q}^{(1) e e 31}$ exists and is equal to 1 as an example, and we multiply the ratio of cross sections of other parameters to get the corresponding effective cross sections.

\section{Backgrounds at the $\mathrm{LHeC}$}

The dominant SM background is made up of inclusive processes which contain the corresponding final states: $\ell^{+}+\ell^{-}+E_{T}^{\text {miss }}+b / \bar{b} / j$. At the $\mathrm{LHeC}$, there are three dominant backgrounds: (a) Vector boson fusion processes at the $\mathrm{LHeC}$ can introduce the same final states, and the $b$ jet is coming from the scattering of the initial proton beam; (b) inclusive top-quark production processes at the $\mathrm{LHeC}$ can lead to similar signatures from their top decays (they are actually quite large as their cross section reaches $3.5 \mathrm{pb}$ at our setup at the $\mathrm{LHeC}$ ); (c) processes including $Z$ bosons can have rich leptonic and hadronic final states and thus can be sources of background. The above backgrounds can be summarized as

(a) $e^{-} p \rightarrow l^{+}+e^{-}+E_{T}^{\text {miss }}+b / \bar{b} / j$ or $e^{-} p \rightarrow l^{-}+e^{-}+$ $E_{T}^{\text {miss }}+b / \bar{b} / j$

(b) $e^{-} p \rightarrow \bar{t}+$ all or $e^{-} p \rightarrow \bar{t}+$ all + all or $e^{-} p \rightarrow \bar{t}+$ $t+$ all + all,

(c) $e^{-} p \rightarrow e^{-}+z+z+j$ or $e^{-} p \rightarrow E_{T}^{\text {miss }}+z+z+j$ or $e^{-} p \rightarrow E_{T}^{\text {miss }}+z+j$ or $e^{-} p \rightarrow e^{-}+z+j$.

Note that all inclusive processes containing more jets for the mentioned background are negligible.

In the next subsection, we show that all the above background can be effectively removed using several kinematical cuts listed in the following. This is mainly due to the requirement of the final states to be from single top decays. The rich sources of the leptonic final states in the background make it possible to distinguish signal from background by requiring an exact number of leptons to be the decay products of single top and $W$ bosons.

\section{Kinematical cuts}

In order to distinguish signal from background, we put kinematical cuts on both the signal and background:

(i) Cut I: Requiring exactly one electron, one positron, one $E_{T}^{\text {miss }}$ and only one $b$-tagged jet with a minus charge as the $b$ jet is coming from the massive particle $t$.

(ii) Cut II: Based on cut I, requiring the transverse mass $\left|M\left(e^{+}, E_{T}^{\text {miss }}\right)-M_{W}\right|<10 \mathrm{GeV}$.

(iii) Cut III: Based on cut II, requiring the invariant mass $\left|M\left(e^{+}, E_{T}^{\text {miss }}, b\right)-M_{t}\right|<40 \mathrm{GeV}$.

Note here we only consider the $e^{+}+E_{T}^{\text {miss }}+b$ final states of the top quark for simplicity. The antimuon final states can also be considered in future detector simulations. We produced 500 thousand events for each signal and background; the cross section and the efficiency, defined as $\frac{N(\text { after cuts })}{N \text { (initial events })}$, for all signal and background are shown in Table II. From Table II, we can see that cut I is quite efficient at distinguishing between the signal and background even though only $13.3 \%$ [51] of the top quark can have $e^{+}, E_{T}^{\text {miss }}$ at the final states. Cut II is extremely good at distinguishing background containing the top quark and $Z$ boson. For background (b), this is due to the fact that the missing energy and positron in background (b), which mostly contain multiple tops, can have different sources; thus it is not likely for them to reconstruct the $W$-boson mass. As for background (c), this is quite straightforward as it is rare for a $Z$ boson to decay into a $W$ boson. For background (a), however, there are processes that contain $W \rightarrow e^{+} \nu_{e}$, which, as shown in Fig. 2, makes it difficult to separate the background from the signal. Luckily, this background has already been reduced by the requirement of only one $b$-tagged jet of cut I. This is due to the fact that

TABLE II. Cut flows for the signal and background processes, applying the kinematical cuts mentioned in Sec. III D at the LHeC. The efficiency is defined as $N_{\text {after cuts }} / N_{\text {initial events }}$.

\begin{tabular}{lcccc}
\hline \hline Processes & Signal & Background (a) & Background (b) & Background (c) \\
\hline$\sigma(\mathrm{fb})$ & 1630 & 818 & 3520 & 1016 \\
Cut I efficiency & $2.83 \mathrm{e}-02$ & $8.06 \mathrm{e}-04$ & $9.60 \mathrm{e}-03$ & $2.04 \mathrm{e}-03$ \\
Cut II efficiency & $6.28 \mathrm{e}-03$ & $2.88 \mathrm{e}-04$ & $5.85 \mathrm{e}-05$ & $6.00 \mathrm{e}-06$ \\
Cut III efficiency & $2.46 \mathrm{e}-03$ & $1.08 \mathrm{e}-04$ & $1.61 \mathrm{e}-05$ & $2.00 \mathrm{e}-6$ \\
Effective events $\left(2 \mathrm{ab}^{-1}\right)$ & 8020 & 177 & 113 & 4 \\
\hline \hline
\end{tabular}




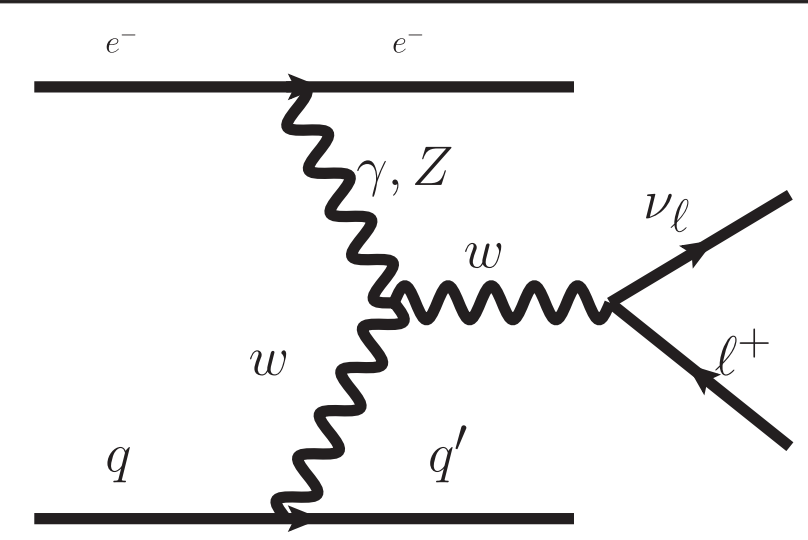

FIG. 2. One example of the background process $e^{-} p \rightarrow l^{+}+$ $e^{-}+\nu_{l}+b$ at LHeC which contains $W$ bosons as a mediator for $l^{+}$and $\nu_{l}$.

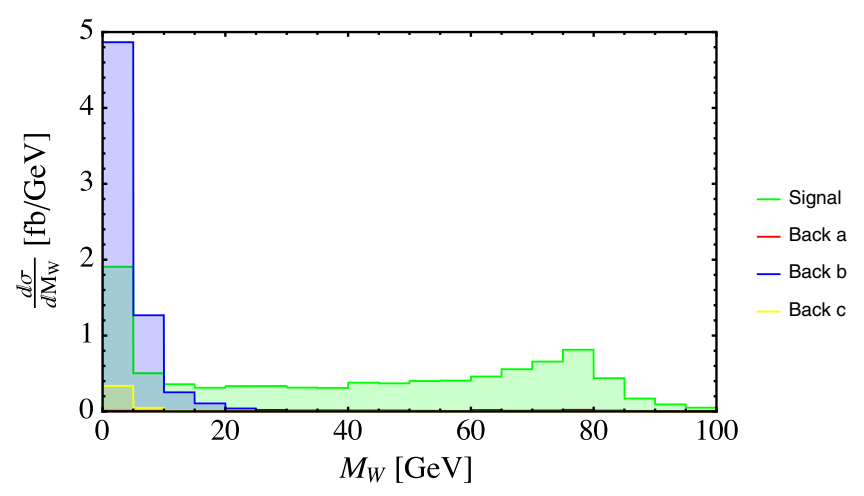

FIG. 3. Reconstructed $W$-boson transverse mass from $e^{+}$and $E_{T}^{\text {miss }}$ after cut I. The green bar presents the signal, while the red, blue and yellow represent backgrounds (a), (b) and (c), respectively. The bin size is $5 \mathrm{GeV}$.

other than those produced by the parenting top quark, the $b$-tagged jets in background (a) are produced by the decay of gluons which are more likely to produce more than one $b$-tagged jet with a minus sign. As we already obtain big enough $S / \sqrt{B}$ from the above cuts, cuts III, which we use to reconstruct the top mass, are quite loose and not very effective. This selection of cuts is also justified in Figs. 3 and 4. Especially in Fig. 4, it is shown that we are able to

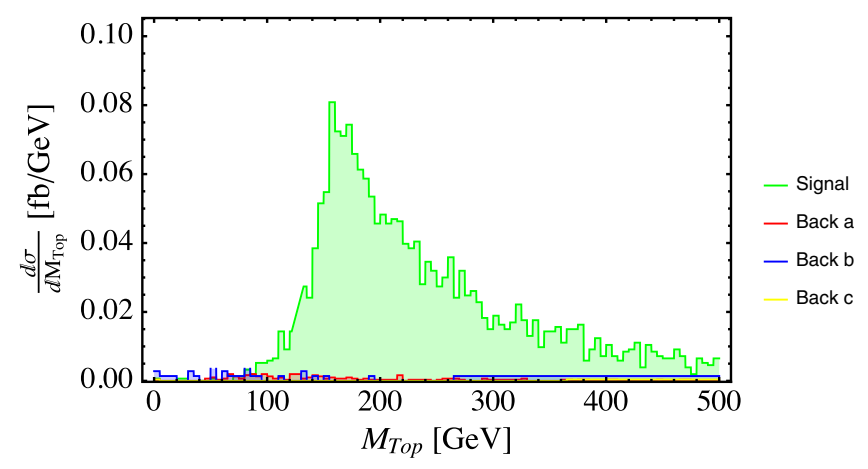

FIG. 4. Reconstructed top mass from $W$ and $b$-tagged jets after cut II. The green bar represents the signal, while the red, blue and yellow represent backgrounds (a), (b) and (c), respectively. The bin size is $5 \mathrm{GeV}$.

get a good distribution of reconstructed top mass with much higher efficiency compared to the background, which indicates that the cuts we applied are efficient at distinguishing the signal from background.

\section{E. Sensitivity limits}

With the cross section and efficiencies calculated above, we then apply a goodness-of-fit with the method of least squares $\left(\chi^{2}\right)$ analysis requiring $\chi^{2}=\left(N_{\text {tot }}-N_{B}\right)^{2} / N_{B}>$ 3.84 at $95 \%$ C.L. [51] at the $\mathrm{LHeC}$ with $2 \mathrm{ab}^{-1}$ luminosity. Thus, the corresponding limits for different parameters in 31 and 13 generations, assuming only one parameter exists, are calculated by multiplying by their own cross sections and are shown in Table III. Summarized in the basis of an independent parameter, we obtain
(i) $\sqrt{\left(V_{L L}^{312}+V_{R R}^{31}{ }^{2}\right)}<6.47 \times 10^{-2}$
(ii) $\sqrt{\left(V_{L R}^{312}+V_{R L}^{312}\right)}<1.09 \times 10^{-1}$
(iii) $\sqrt{S_{R R}^{312}}<2.17 \times 10^{-1}$
(iv) $\sqrt{T_{R R}^{312}}<5.27 \times 10^{-3}$.

The limits for 32 and 23 generations can be obtained by multiplying a simple 32.2 factor, due to the different content of the $u$-quark and $c$-quark in the $\mathrm{LHeC}$ proton beam.

TABLE III. Limits for the corresponding coefficient for the 31 and 13 (32 and 23) generation from the $e^{+}+\nu_{e}$ channel at the LHeC. The limits are calculated at 95\% C.L. for $2 \mathrm{ab}^{-1}$ LHeC.

\begin{tabular}{lccc}
\hline \hline Parameters & $C_{l q}^{(1) e e 31}+$ H.c. & $C_{l q}^{(3) e e 31}+$ H.c. & $C_{e u}^{e e 31}+$ H.c. \\
\hline Limits & $0.0647(0.367)$ & $0.0647(0.367)$ & $0.0647(0.367)$ \\
Parameters & $C_{e q}^{e e 31}+$ H.c. & $C_{l u}^{e e 31}+$ H.c. & $C_{l e q u}^{(1) e e 13}+$ H.c. \\
Limits & $0.109(0.617)$ & $0.109(0.617)$ & $0.217(1.23)$ \\
Parameters & $C_{l e q u}^{(1) e e 31}+$ H.c. & $C_{l e q u}^{(3) e e 13}+$ H.c. & $C_{l e q u}^{(3) e e 31}+$ H.c. \\
Limits & $0.217(1.23)$ & $0.0209(0.102)$ & $0.0209(0.119)$ \\
\hline \hline
\end{tabular}




\section{CONCLUSION}

In this work we have considered the four-fermion top flavor changing neutral current operators in the basis of dimension-six Standard Model effective field theory. We studied the process $e^{-} p \rightarrow e^{-} t$, in particular, at the proposed electron proton collider $\mathrm{LHeC}$, using its signature to set limits for the involved operators in the dimension-six SMEFT. We performed our simulation at the full detector level, requiring $e^{-}+e^{+}+E_{T}^{\text {miss }}+b$ selection specifically at the $\mathrm{LHeC}$. The corresponding backgrounds were reduced by setting kinematical cuts, requiring the reconstructed $W$-boson transverse mass and top-quark mass. The sensitivities at the $2 \mathrm{ab}^{-1} \mathrm{LHeC}$ were calculated by using a goodness-of-fit with the method of least squares $\left(\chi^{2}\right)$ analysis. We obtained upper limits for $C_{l q}^{(1) e e 31}, C_{l q}^{(3) e e 31}$, $C_{e u}^{e e 31}+$ H.c. $<0.0647, C_{e q}^{e e 31}, C_{l u}^{e e 31}+$ H.c. $<0.109, C_{\text {lequ }}^{(1) e e 13}$, $C_{\text {lequ }}^{(1) e e 31}+$ H.c. $<0.217$ and $C_{\text {lequ }}^{(3) e e 13}, \quad C_{\text {lequ }}^{(3) e e 31}+$ H.c. $<$ 0.0209 assuming only one operator exists in the dimension-six SMEFT for couplings of quarks at the first and third generations. These limits were calculated at 95\% C.L. for $2 \mathrm{ab}^{-1}$ LHeC. The couplings between quarks in the second and third generations can be obtained similarly. However, this process has no sensitivities for the couplings between two third generations as $e^{-} t \rightarrow e^{-} t$ is negligible. In other words, we have already obtained competitive limits in flavor changing four-fermion operators such as $C_{l q}^{(1,3)}$ and $C_{e q}$ at the LHeC using a simple process. We have also gained new limits on the operators, including right-handed singlets of quarks such as $C_{l u}, C_{e u}$, $C_{\text {lequ }}^{(1,3)}$, which are not covered in the $B$ flavor physics.

These direct limits are already comparable to the indirect limits such as precise measurements of $B$ physics at the LHC through the CKM matrix. Note that $e^{+}, e^{-}$colliders are expected to have even cleaner background, thus producing indirect upper limits which are about a magnitude smaller for the operators.

\section{ACKNOWLEDGMENTS}

W. L. is supported by the China Scholarship Council (Grant CSC No. 2016 08060325). H. S. is supported by the National Natural Science Foundation of China (Grant No. 11675033) and by the Fundamental Research Funds for the Central Universities (Grant No. DUT18LK27).
[1] C. Zhang, Chin. Phys. C 42, 023104 (2018).

[2] A. Buckley, C. Englert, J. Ferrando, D. J. Miller, L. Moore, M. Russell, and C.D. White, J. High Energy Phys. 04 (2016) 015.

[3] A. Buckley, C. Englert, J. Ferrando, D. J. Miller, L. Moore, M. Russell, and C.D. White, Phys. Rev. D 92, 091501 (2015).

[4] C. Zhang and S. Willenbrock, Phys. Rev. D 83, 034006 (2011).

[5] D. Barducci et al., arXiv:1802.07237.

[6] M. Chala, J. Santiago, and M. Spannowsky, J. High Energy Phys. 04 (2019) 014.

[7] N. P. Hartland, P. Nathan, F. Maltoni, E. R. Nocera, J. Rojo, E. Slade, E. Vryonidou, and C. Zhang, J. High Energy Phys. 04 (2019) 100.

[8] B. Grzadkowski, M. Iskrzynski, M. Misiak, and J. Rosiek, J. High Energy Phys. 10 (2010) 085.

[9] A. Dedes, W. Materkowska, M. Paraskevas, J. Rosiek, and K. Suxho, J. High Energy Phys. 06 (2017) 143.

[10] A. Dedes, M. Paraskevas, J. Rosiek, K. Suxho, and L. Trifyllis, arXiv:1904.03204

[11] S. L. Glashow, J. Iliopoulos, and L. Maiani, Phys. Rev. D 2 , 1285 (1970).

[12] O. Bruening and M. Klein, Mod. Phys. Lett. A 28, 1330011 (2013).

[13] J. L. A. Fernandez et al. (LHeC Study Group), J. Phys. G 39, 075001 (2012).
[14] B. Capdevila, A. Crivellin, S. Descotes-Genon, J. Matias, and J. Virto, J. High Energy Phys. 01 (2018) 093.

[15] W. Altmannshofer and D. M. Straub, Eur. Phys. J. C 75, 382 (2015).

[16] D. Ghosh, M. Nardecchia, and S. A. Renner, J. High Energy Phys. 12 (2014) 131.

[17] A. Greljo and D. Marzocca, Eur. Phys. J. C 77, 548 (2017).

[18] M. Jung and D. M. Straub, J. High Energy Phys. 01 (2019) 009.

[19] S. Bar-Shalom and J. Wudka, Phys. Rev. D 60, 094016 (1999).

[20] H. Abramowicz et al. (CLICdp Collaboration), arXiv: 1807.02441.

[21] A. Escamilla, A. O. Bouzas, and F. Larios, Phys. Rev. D 97, 033004 (2018).

[22] G. Durieux, Proc. Sci., DIS2017 (2018) 088 [arXiv:1708 .09849].

[23] L. Shi, andC. Zhang, arXiv:1906.04573

[24] G. Durieux, M. Perelló, M. Vos, and C. Zhang, J. High Energy Phys. 10 (2018) 168.

[25] S. Behera, R. Islam, M. Kumar, P. Poulose, and R. Rahaman, arXiv:1811.04681.

[26] O. Cakir, A. Yilmaz, I. T. Cakir, A. Senol, and H. Denizli, Nucl. Phys. B944, 114640 (2019).

[27] I. T. Cakir, A. Yilmaz, H. Denizli, A. Senol, H. Karadeniz, and O. Cakir, Adv. High Energy Phys. 2017, 1572053 (2017). 
[28] X. Wang, H. Sun, and X. Luo, Adv. High Energy Phys. 2017, 4693213 (2017).

[29] H. Sun and X. Wang, Eur. Phys. J. C 78, 281 (2018).

[30] W. Liu, H. Sun, X. Wang, and X. Luo, Phys. Rev. D 92 , 074015 (2015).

[31] H. Hesari, H. Khanpour, and M. M. Najafabadi, Phys. Rev. D 97, 095041 (2018).

[32] L. Duarte, G. A. González-Sprinberg, and O. A. Sampayo, Phys. Rev. D 91, 053007 (2015).

[33] J. Alcaide, S. Banerjee, M. Chala, and A. Titov, arXiv: 1905.11375

[34] I. A. Sarmiento-Alvarado, A. O. Bouzas, and F. Larios, J. Phys. G 42, 085001 (2015).

[35] M. Russell, Top Quark Physics in the Large Hadron Collider Era, Ph.D. thesis, Glasgow University, 2017.

[36] A. Cerri et al., arXiv:1812.07638.

[37] V. Cirigliano, M. Gonzalez-Alonso, and M. L. Graesser, J. High Energy Phys. 02 (2013) 046.

[38] J. Aebischer, A. Crivellin, M. Fael, and C. Greub, J. High Energy Phys. 05 (2016) 037.

[39] D. Buttazzo, A. Greljo, G. Isidori, and D. Marzocca, J. High Energy Phys. 11 (2017) 044.

[40] A. Alloul, N. D. Christensen, C. Degrande, C. Duhr, and B. Fuks, Comput. Phys. Commun. 185, 2250 (2014).
[41] C. Degrande, C. Duhr, B. Fuks, D. Grellscheid, O. Mattelaer, and T. Reiter, Comput. Phys. Commun. 183, 1201 (2012).

[42] J. Alwall, R. Frederix, S. Frixione, V. Hirschi, F. Maltoni, O. Mattelaer, H. S. Shao, T. Stelzer, P. Torrielli, and M. Zaro, J. High Energy Phys. 07 (2014) 079.

[43] T. Sjostrand, S. Mrenna, and P. Z. Skands, J. High Energy Phys. 05 (2006) 026.

[44] S. Ovyn, X. Rouby, and V. Lemaitre, arXiv:0903.2225.

[45] M. Dobbs and J. B. Hansen, Comput. Phys. Commun. 134, 41 (2001).

[46] M. Klein, LHeC detector design, 25th International Workshop on Deep Inelastic Scattering, Birmingham (2017), https://indico.cern.ch/event/568360/contributions/2523637/.

[47] G. Azuelos, H. Sun, and K. Wang, Phys. Rev. D 97, 116005 (2018).

[48] M. Klein, U. Klein, P. Kostka, and S. Kawaguchi. (private communication).

[49] C. S. Deans (NNPDF Collaboration), in Proceedings, 48th Rencontres de Moriond on QCD and High Energy Interactions: La Thuile, Italy, 2013 (2013), pp. 353-356, https://inspirehep.net/record/1227810.

[50] R. D. Ball et al., Nucl. Phys. B867, 244 (2013).

[51] C. Patrignani et al. (Particle Data Group), Chin. Phys. C 40, 100001 (2016).

[52] C. Degrande, J. Phys. Conf. Ser. 452, 012009 (2013). 